NIST Technical Note 1876

\title{
MicroChar \\ An Application for Quantitative Analysis of Cement and Clinker Microstructure Images
}

\author{
Jeffrey W. Bullard
}

This publication is available free of charge from:

http://dx.doi.org/10.6028/NIST.TN.1876 
NIST Technical Note 1876

\title{
MicroChar \\ An Application for Quantitative Analysis of Cement and Clinker Microstructure Images
}

\author{
Jeffrey W. Bullard \\ Materials and Structural Systems Division \\ Engineering Laboratory
}

This publication is available free of charge from: http://dx.doi.org/10.6028/NIST.TN.1876

April 2015

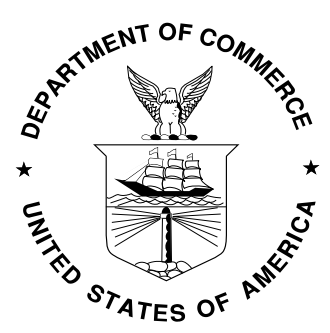

U.S. Department of Commerce

Penny Pritzker, Secretary

National Institute of Standards and Technology

Willie May, Acting Under Secretary of Commerce for Standards and Technology and Acting Director 
Certain commercial entities, equipment, or materials may be identified in this document in order to describe an experimental procedure or concept adequately. Such identification is not intended to imply recommendation or endorsement by the National Institute of Standards and Technology, nor is it intended to imply that the entities, materials, or equipment are necessarily the best available for the purpose.

National Institute of Standards and Technology Technical Note 1876

Natl. Inst. Stand. Technol. Tech. Note 1876, 20 pages (April 2015) CODEN: NTNOEF

This publication is available free of charge from: http://dx.doi.org/10.6028/NIST.TN.1876 


\begin{abstract}
Accurate quantitative data on cement and clinker microstructure images can prove valuable for monitoring and controlling the manufacturing of cement-based powders. Furthermore, quantitative characterization of microstructure is an essential input to microstructure-based computer models of cementitious material processing and properties. This document describes the use and operating principles of MicroChar, a computer application for automatically calculating a range of microstructural properties from an indexed 2D image. Among the properties calculated are the volume fraction, mass fraction, and surface area fraction of each phase in the image, as well as two-point correlation functions for quantifying the spatial distribution of the phases throughout the structure. The application also enables the user to package the data obtained on cement powders for uploading to the Virtual Cement and Concrete Testing Laboratory Consortium (VCCTL) software.
\end{abstract}

Keywords: Building technology; cement; clinker; microstructure; computer modeling 


\section{Contents}

1 Introduction 1

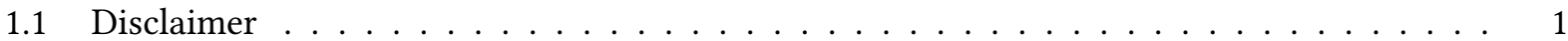

1.2 Intent and Use $\ldots \ldots \ldots \ldots \ldots \ldots \ldots \ldots \ldots \ldots$

1.3 Notation Conventions . . . . . . . . . . . . . . . . . . . . . . . . . 1

2 Software Prerequisites 2

3 Installation $\quad 2$

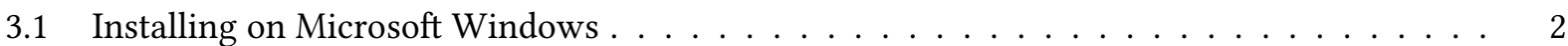

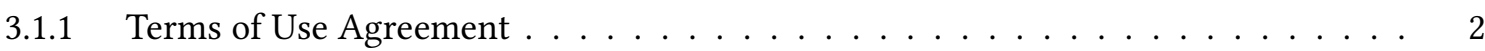

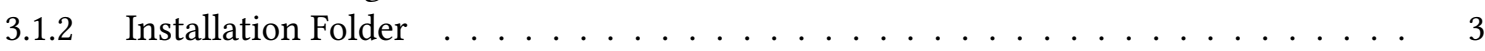

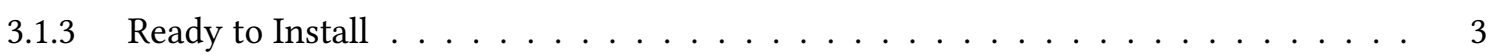

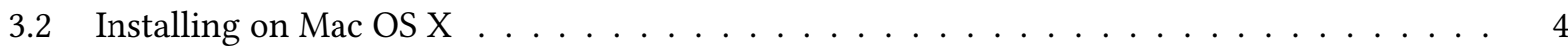

4 Uninstalling MicroChar 4

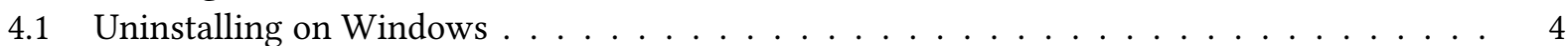

4.2 Uninstalling on Mac OS $\mathrm{X} \ldots \ldots \ldots \ldots \ldots \ldots$

5 Using MicroChar $\quad 5$

5.1 Image Requirements . . . . . . . . . . . . . . . . . . . . . . . . . 5

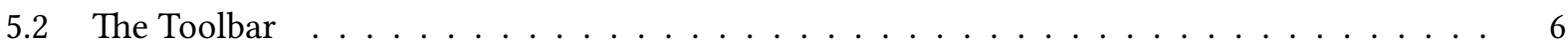

5.3 Cement Powder Images . . . . . . . . . . . . . . . . . . . . . . . . 7

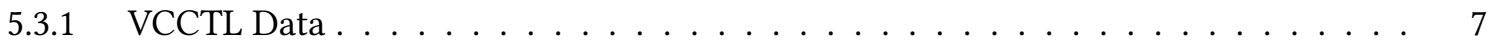

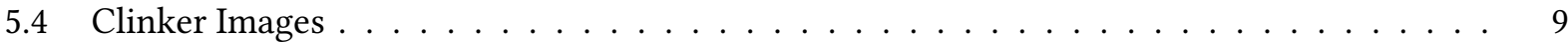

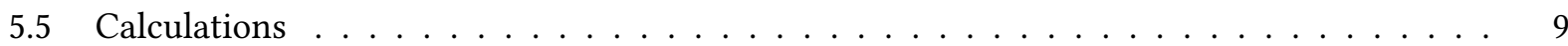

6 Calculation Principles and Algorithms $\quad 11$

6.1 Volume Fraction . . . . . . . . . . . . . . . . . . . . . . . . . 11

6.2 Mass Fraction $\ldots \ldots \ldots \ldots \ldots \ldots \ldots \ldots \ldots \ldots$

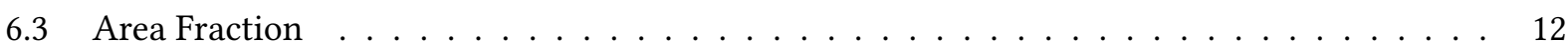

6.4 Two-point correlation functions $\ldots \ldots \ldots \ldots \ldots \ldots \ldots \ldots$

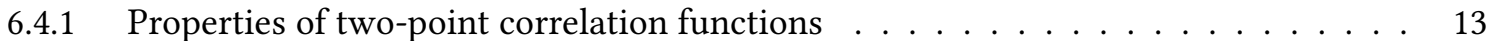

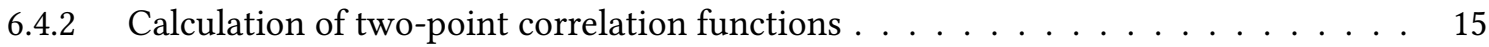

7 Summary $\quad 15$

$\begin{array}{ll}\text { References } & 15\end{array}$ 


\section{Introduction}

This document provides guidance for using MicroChar, subject to Disclaimer in Section 1.1, a computer application for performing quantitative analysis of cement and clinker microstructural images. The application consists of a graphical user interface and the underlying computer models and programs that allow users to open indexed microstructure images, to identify the constituent mineral phases, to determine mass, volume, and surface area fractions of those phases, and to characterize the spatial distribution of those phases in terms of two-point correlation functions. Optionally, for cement powder microstructure images, the user may provide additional information and package the resulting data in a collection of files suitable for direct uploading to the Virtual Cement and Concrete Testing Laboratory 9.5 (VCCTL) software platform.

\subsection{Disclaimer}

This software was developed at the National Institute of Standards and Technology (NIST) by employees of the Federal Government in the course of their official duties. Pursuant to Title 17 Section 105 of the United States Code this software is not subject to copyright protection and is in the public domain. NIST assumes no responsibility whatsoever for the use of MicroChar by other parties, and makes no guarantees, expressed or implied, about its quality, reliability, or any other characteristic. NIST would appreciate acknowledgment, such as an appropriate citation in a publication, if the software is used.

The U.S. Department of Commerce makes no warranty, expressed or implied, to users of MicroChar, and accepts no responsibility for its use. Users of MicroChar assume sole responsibility under Federal law for determining the appropriateness of its use in any particular application; for any conclusions drawn from the results of its use; and for any actions taken or not taken as a result of analyses performed using these tools. All results should be critically evaluated by an informed user.

\subsection{Intent and Use}

The computer program described in this guide constitutes a prototype application for quantitative image analysis of cement powder and clinker microstructures. It has been compiled from the best knowledge and understanding currently available, but has some limitations that must be understood and considered by the user. MicroChar is intended for use by persons competent in the field of cement-based materials and having some familiarity with computers.

\subsection{Notation Conventions}

Throughout this guide, the following text conventions will be used:

- italicized text will indicate

- words on a MicroChar window (for example, the Execute button);

- key combinations Ctrl-O to indicate the keyboard combination of Control plus O).

- Courier font will be used to indicate

- a file name: mycement.img or disrealnew. exe;

- the location of a folder or file: C: "Users"user"mycement; 
- web addresses: http://oracle.com

- Bold text will indicate an important note or warning that the user must heed (for example, Beware that calculating correlation functions can take a considerable amount of time).

- Words in all capital letters will be used to denote variables in file names or user names for which the user must supply the correct value.

\section{Software Prerequisites}

The following requirements must be met for successful installation and running of MicroChar:

- Operating system: Microsoft Windows $7^{*}$ or Mac OS X $10.9^{*}$ or later. Other versions of Windows, such as XP, Vista, or Windows 8, or earlier versions of Mac OS X may or may not be compatible with MicroChar.

- A minimum of $2 \mathrm{~GB}$ RAM and minimum free hard disk space of 1 GB for long-term use of the software.

\section{Installation}

\subsection{Installing on Microsoft Windows}

To install MicroChar on Windows, download and then double click on the installer file (Microchar1.0-windows-installer. exe) icon to the Desktop. A series of windows will guide you through the installation process.

\subsubsection{Terms of Use Agreement}

This dialog requires you to acknowledge the software disclaimer, which has language identical to that in Section 1.1. If you accept the disclaimer agreement, you can proceed with the installation by pressing the Next button. Otherwise, the installation will be cancelled.

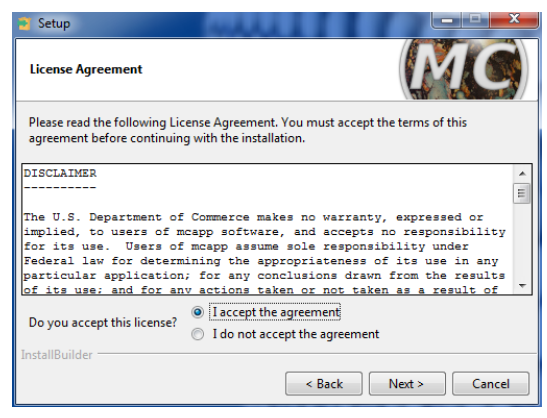

${ }^{*}$ Certain commercial equipment and/or materials are identified in this report in order to adequately specify the experimental procedure. In no case does such identification imply recommendation or endorsement by the National Institute of Standards and Technology, nor does it imply that the equipment and/or materials used are necessarily the best available for the purpose. 


\subsubsection{Installation Folder}

You may install MicroChar in any location on your computer, provided that you have the adequate read/write permissions. The default path and folder is MicroChar located in C: "Program Files. You may accept that default or change it to another location. However, the final folder on the path must be named MicroChar The software may not work properly if it is not installed in a folder named MicroChar.

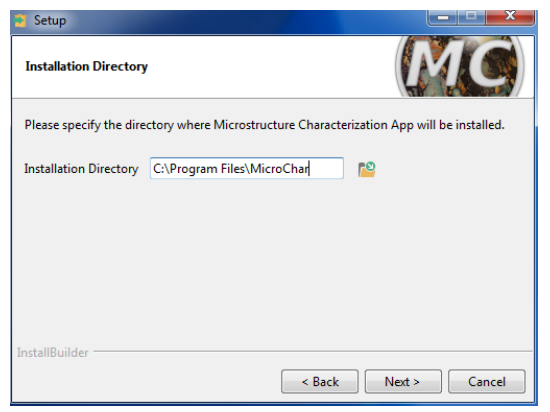

\subsubsection{Ready to Install}

The next dialog box indicates that the software is ready to install. Click the Next button to begin the installation.

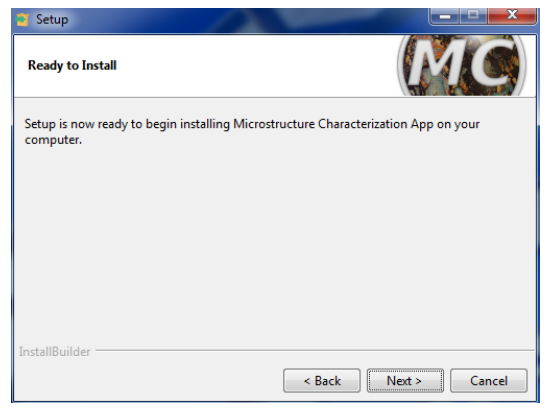

The installation should finish within a few seconds, after which a window will notify you that the installation is complete. Close this window by clicking on the Finish button.

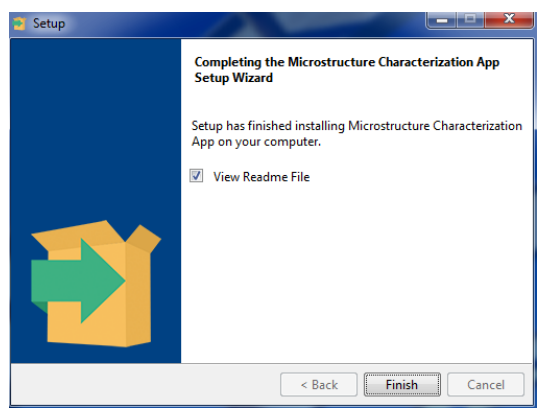

When the installation is complete, a new shortcut icon for MicroChar should appear on your Desktop, and MicroChar should also be listed in the Start Menu. Either one can be used to launch the application. 


\subsection{Installing on Mac OS X}

Double click the compressed disk image file, Microchar.dmg, which will mount the disk image and open a window on your Desktop. The application may be installed anywhere by dragging the circular application icon to a file folder. A shortcut to the Applications folder is provided in the mounted disk image for convenience.

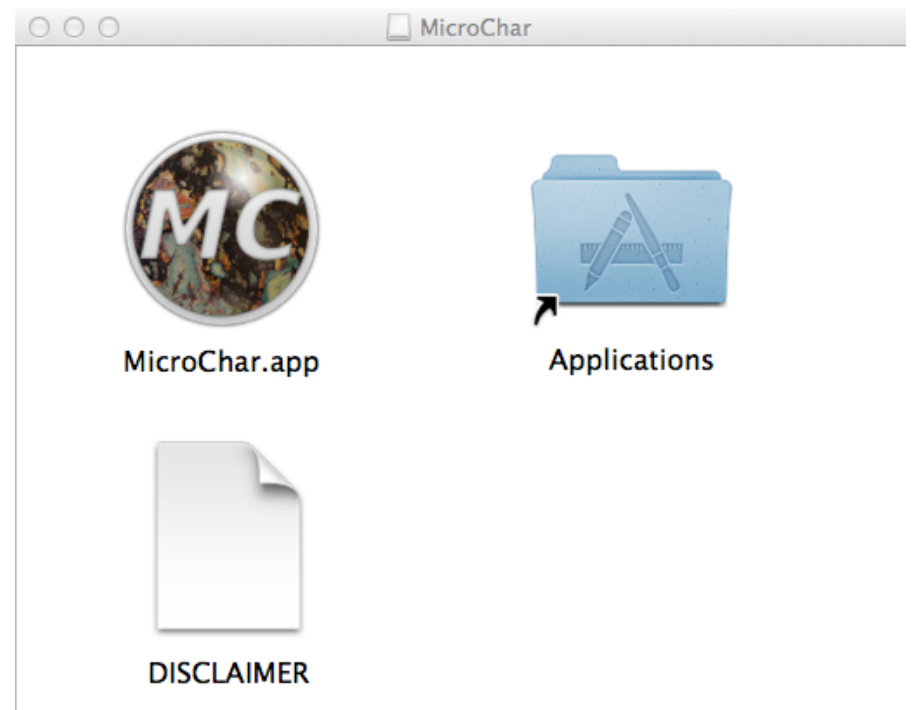

Once the application is installed, eject the disk image by locating its icon on the Desktop and dragging it to the Trash on the Dock.

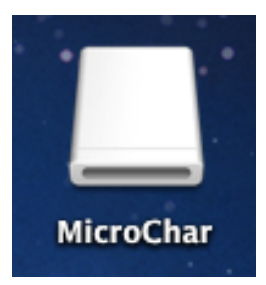

\section{Uninstalling MicroChar}

\subsection{Uninstalling on Windows}

The easiest way to remove from a Windows computer is to launch the uninstaller, which can be found in the Start Menu.

Maintenance

MicroChar

Uninstall Microstructure Characteriz

Microsoft Office 2013 
A dialog box will ask if you are sure you want to uninstall the application. Click Yes to start removing the software. The entire process should finish within a few seconds, after which another window will appear to announce that the removal is complete. This removes all the installed files, including the Desktop shortcut icon and the Start Menu items.
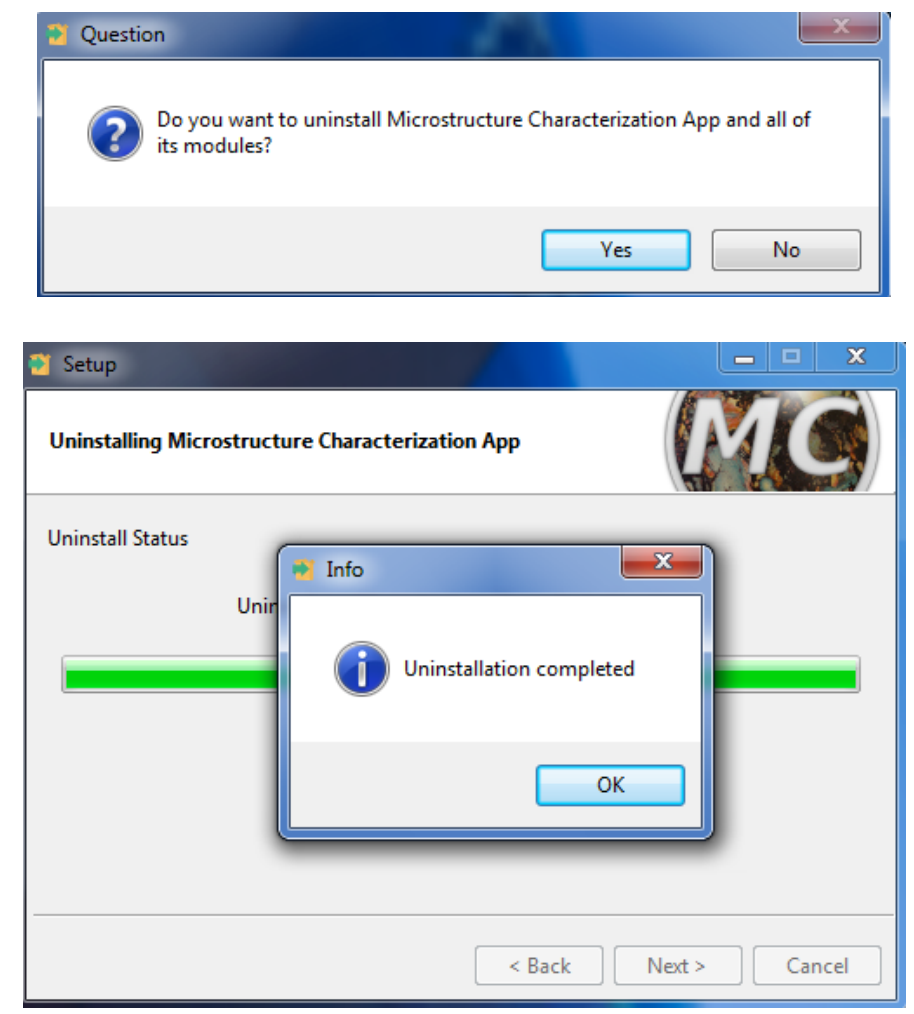

\subsection{Uninstalling on Mac OS $\mathrm{X}$}

Drag the application from its installed folder to the Trash to remove it from a Mac OS X computer.

\section{Using MicroChar}

\subsection{Image Requirements}

MicroChar works with a 2D indexed image file. The details of preparaing such an image file from scanning electron microscope data are beyond the scope of this document. Instructions for doing so can be found in a recent report [1]. The indexed image must meet the following formatting requirements:

1. The file must contain ASCII text.

2. There must be no header information of any kind in the image.

3. Each row of the image corresponds to one line of text in the file, uninterrupted by new lines or carriage returns.

4. The number of characters in a single line corresponds to the number of columns in the $2 \mathrm{D}$ image. 
5. Only tab characters or spaces should separate the characters within a line.

6. Each character in a line must be a positive integer that can be used to uniquely identify the phase located in the pixel at the corresponding row and column of the image.

Images meeting these requirements can be readily produced using ImageJ, a freely available image processing software package [2]. In addition to the formatting requirements, the image resolution (in units of $\mu \mathrm{m} / \mathrm{pixel}$ ) should be known.

Launch MicroChar by double clicking its icon, which will open the main window.

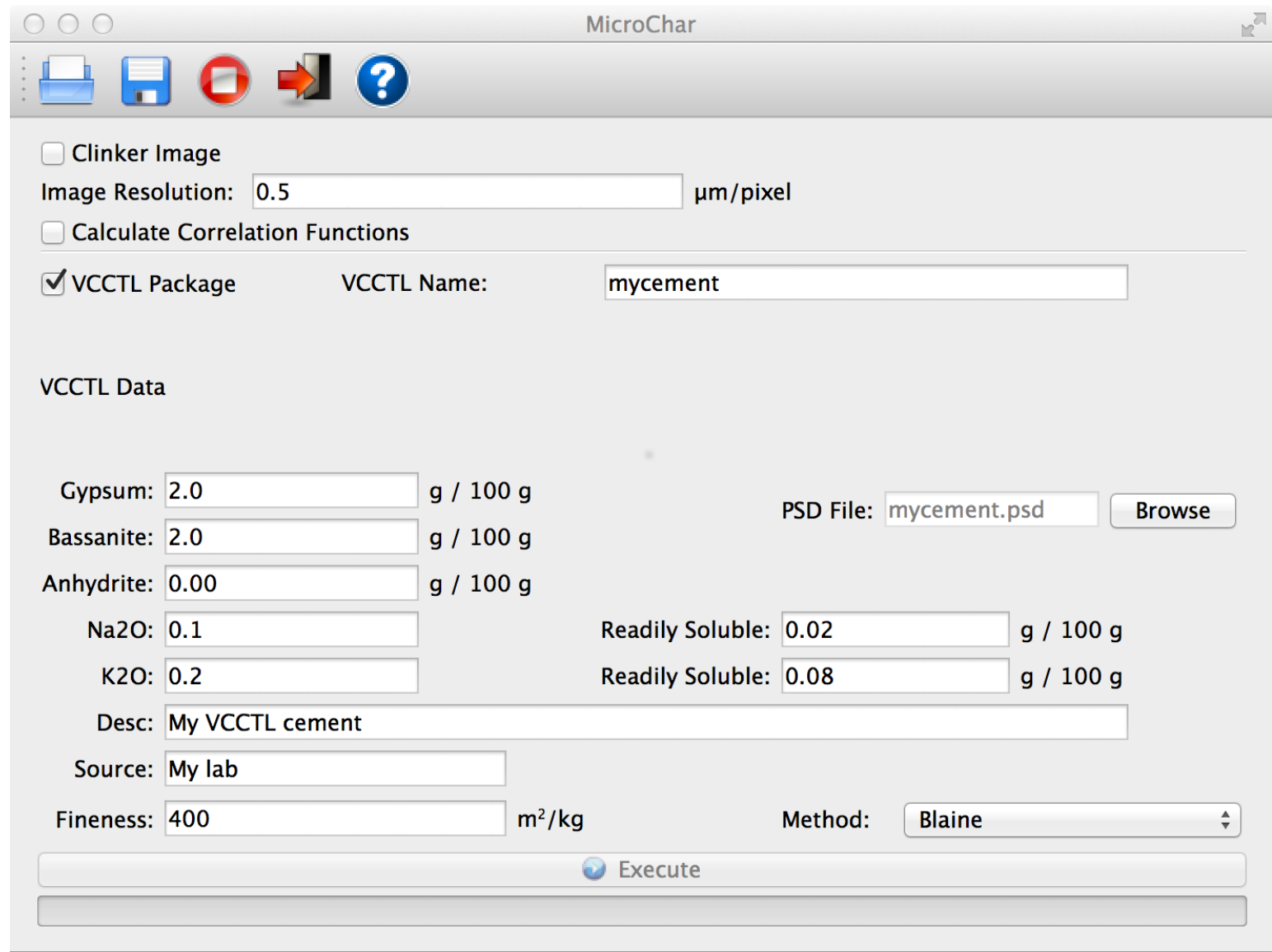

Mousing over an item in the window will display its tooltip. For more context-specific information, click on the blue question mark icon in the toolbar and then click on the window item to reveal more information about it.

\subsection{The Toolbar}

The toolbar at the top of the main window provides quick access to common tasks, as described in Table 1.

To begin a session, open the indexed image using the toolbar (or type $\mathrm{Ctrl}-\mathrm{O}$ ), and navigate to the desired image file. The application works equally well on indexed images either of clinker or of powdered cement embedded in epoxy, but it analyzes the void space and identifies perimeteris differently for the two kinds of images. 
Table 1: Toolbar actions.

\begin{tabular}{cc} 
Action & Keyboard Shortcut \\
\hline & $\begin{array}{c}\text { Open an indexed image } \\
\text { Save calculations and } \\
\text { associated files } \\
\text { Interrupt a calculation } \\
\text { and erase intermediate } \\
\text { data files }\end{array}$ \\
$\begin{array}{l}\text { Close the main window } \\
\text { and exit the application } \\
\text { Obtain context-specific } \\
\text { information about any } \\
\text { item by clicking on it }\end{array}$ & Ctrl-Q \\
\hline
\end{tabular}

\subsection{Cement Powder Images}

For cement powder images, ensure that the Clinker Image checkbox is unchecked. Specify the image resolution in the text field, in terms of the width of a single pixel in micrometer $(\mu \mathrm{m})$ units. The pixel dimension should be known at the time that the microstructure image is acquired experimentally, and is necessary to associate a length scale with the two-point correlation functions that will be calculated if the corresponding checkbox is activated below the resolution text field. Note: Calculating correlation functions can take considerable time, up to $30 \mathrm{~min}$ or more depending on how many phases are in the image.

The remaining items in the window, which are disabled by default, are related to data needed to create a cement data package for the Virtual Cement and Concrete Testing Laboratory (VCCTL) modeling software [3, 4]. Activating the VCCTL Package checkbox will enable these fields, and during the calculations it will prepare a data folder that can be directly uploaded in VCCTL 9.5 to add a new cement for modeling.

\subsubsection{VCCTL Data}

For preparing VCCTL cement data packages, enter the appropriate information about the cement powder in the following data fields:

- VCCTL Name. Enter a name for the cement, which will be assigned to the VCCTL data package folder and all the files therein.

- Gypsum. Enter the mass percent of gypsum (calcium sulfate dihydrate) in the cement, on a total solids basis.

- Bassanite. Enter the mass percent of bassanite (calcium sulfate hemihydrate) in the cement, on a total solids basis. 
- Anhydrite. Enter the mass percent of soluble anhydrite (calcium sulfate) in the cement, on a total solids basis.

- PSD File. Enter the full path and file name of an ASCII text file containing the particle size distribution data for the cement. Use the Browse button to navigate to the approriate file. The file must be formatted as two-column ASCII text, with the left column being the particle diameter in $\mu \mathrm{m}$ and the right column being the probability density function (differential volume fraction for the corresponding size bin). A tab or space(s) may separate the two columns. If a suitable file is not available, a default file will be created and stored for the cement. It can be edited later.

- Na2O/Readily Soluble. Enter the mass percent of sodium oxide in the cement, as determined by X-ray fluorescence spectroscopy, on a total solids basis. In the text field to the right, enter the mass percent of sodium (assumed to be sodium oxide) that is readily soluble, on a total solids basis.

- K2O/ReadilySoluble. Enter the mass percent of potassium oxide in the cement, as determined by $\mathrm{X}$-ray fluorescence spectroscopy, on a total solids basis. In the text field to the right, enter the mass percent of potassium (assumed to be potassium oxide) that is readily soluble, on a total solids basis.

- Desc. Enter a brief description of the type of cement, its application, date received, or other pertinent information.

- Source. Enter a brief description of the source of the material.

- Fineness. Enter the powder's specific surface area $\left(\mathrm{m}^{2} \mathrm{~kg}^{-1}\right)$.

- Method. Select the experimental method used to measure the powder's specific surface area:

- Blaine (air permeability test)

- PSD Analysis (inference from particle size distribution assuming spherical particles)

- BET Analysis (multipoint nitrogen adsorption isotherms)

- Other

Calculations begin by clicking the Execute button at the bottom of the window. The program reads the image file and records all the phase index numbers. When these have been collected, the user is prompted to associate each index with a known phase. A default collection is offered, but each phase can be manually assigned by selecting the desired phase from the pulldown menu next to each phase id found in the image. Click the $O K$ button when the desired selections have been made.

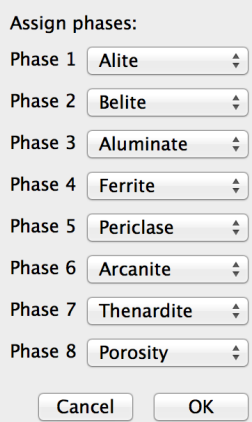


Remaining calculations will be automatically performed. The progress is displayed approximately in the progress bar beneath the Execute button, and relevant messages about the calculations are displayed in the status bar at the bottom of the window. When the calculations are finished, the status bar will provide a text prompt to save the work. Note: If a VCCTL package is requested, the program will calculate required two-point correlation functions for the clinker minerals, whether or not the Calculate Correlation Functions checkbox is activated. However, the VCCTL correlation functions cover a much smaller domain and do not take more than a few seconds each to calculate.

Saving to a user-defined data folder is done by clicking on the blue diskette icon in the toolbar (or typing $(t r l-S$ ), and then navigating to the desired location and typing the name of a new data folder to store the results.

\subsection{Clinker Images}

Activate the Clinker image checkbox only if the opened image is a clinker nodule; otherwise leave it unchecked. Specify the image resolution in the text field, in terms of the width of a single pixel in micrometer $(\mu \mathrm{m})$ units. The pixel dimension should be known at the time that the microstructure image is acquired experimentally, and is necessary to associate a length scale with the correlation functions that will be calculated if the corresponding checkbox is activated. Clinker images cannot be prepared as a VCCTL package, so the rest of the data items in the window can be skipped.

Calculations begin by clicking the Execute button at the bottom of the window. In the same manner as for cement images already described, the program reads the image file, records the phase index numbers, and provides a dialog to prompt for the phase associated with each number. Click the $O K$ button when the desired selections have been made.

\subsection{Calculations}

MicroChar performs the following sequence of operations on both clinker and cement powder images:

1. Small, isolated voids and cracks within grains are removed so that they will not falsely contribute to the perimeter of any phase (clinker images only). ${ }^{\dagger}$

2. The area of each phase is calculated and normalized by the total solid area to yield its area fraction on a total solids basis.

3. The mass fraction of each phase, on a total solids basis, is calculated from the area fractions and knowledge of the density of each phase.

4. The perimeter of each phase is calculated. For clinker images, the perimeter is defined as the boundary of the given phase with any other phase; for cement images, the perimeter is defined as the boundary of the given phase only with interparticle porosity, because such boundaries determine the reactive surface area with water.

5. False color PNG images are created of (a) the microstructure and (b) the phase perimeters.

6. Two-point correlation functions, also known as autocorrelation functions in the field of signal processing, are calculated for each phase (only if the checkbox was activated).

\footnotetext{
$\dagger$ Such "internal" voids and cracks might actually bridge to the surface in the direction perpendicular to the image field, but in the absence of further information we treat them as wholly interior to the grain.
} 
7. The program displays a window summarizing the results of the calculations for each phase.

\begin{tabular}{|llll|}
\hline NAME & MASS & VOLUME & AREA \\
Alite & 0.4609 & 0.4741 & 0.2245 \\
Belite & 0.3479 & 0.3503 & 0.2971 \\
Aluminate & 0.0243 & 0.0264 & 0.0974 \\
Ferrite & 0.1202 & 0.1064 & 0.2617 \\
Arcanite & 0.0039 & 0.0049 & 0.0412 \\
Thenardite & 0.0017 & 0.0021 & 0.0077 \\
Periclase & 0.0411 & 0.0359 & 0.0705 \\
& & & \\
& & & Close \\
\hline
\end{tabular}

8. The program halts and prompts you to save your work if you wish, which you may do by clicking on the disk icon in the tool bar or by typing Ctrl-S.

After saving your work, at least four files are always placed in the saved folder:

1. A file with extension 'pfc' the microstructure on a total solids basis;

\begin{tabular}{lccc} 
& \multicolumn{3}{c}{$\bigcirc$ SRM2686.txt.pfc - Edited } \\
\hline NAME & MASS & VOLUME & AREA \\
& & & \\
Alite & 0.4609 & 0.4741 & 0.2245 \\
Belite & 0.3479 & 0.3503 & 0.2971 \\
Aluminate & 0.0243 & 0.0264 & 0.0974 \\
Ferrite & 0.1202 & 0.1064 & 0.2617 \\
Arcanite & 0.0039 & 0.0049 & 0.0412 \\
Thenardite & 0.0017 & 0.0021 & 0.0077 \\
Periclase & 0.0411 & 0.0359 & 0.0705
\end{tabular}

2. One file with extension 'png' and another with extension 'surf.png'. These are the color PNG images, the first showing the microstructure and second showing the phase perimeters in outline.
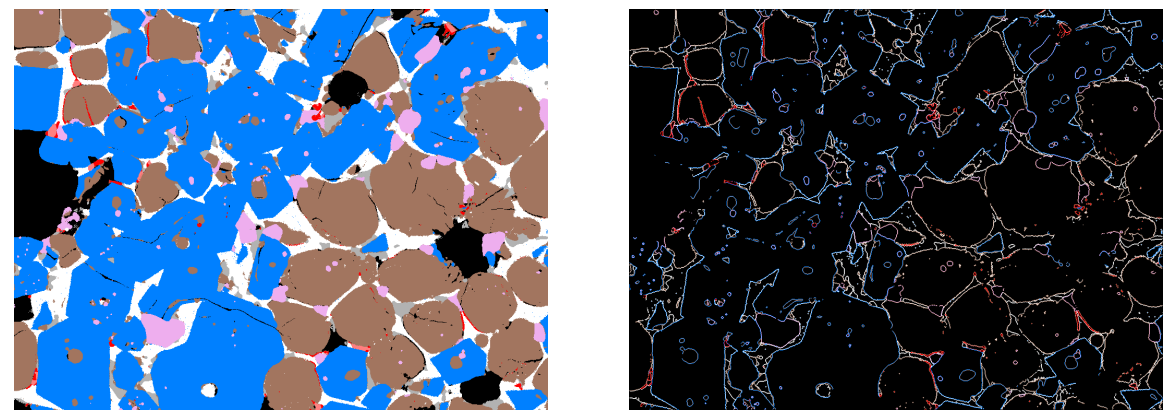

\footnotetext{
†The extension 'pfc' is an acronym for phase fractions of cement (or clinker).
} 
3. A file named Colorkey.png, which displays a key to the different colors in the microstructure images created by the program.

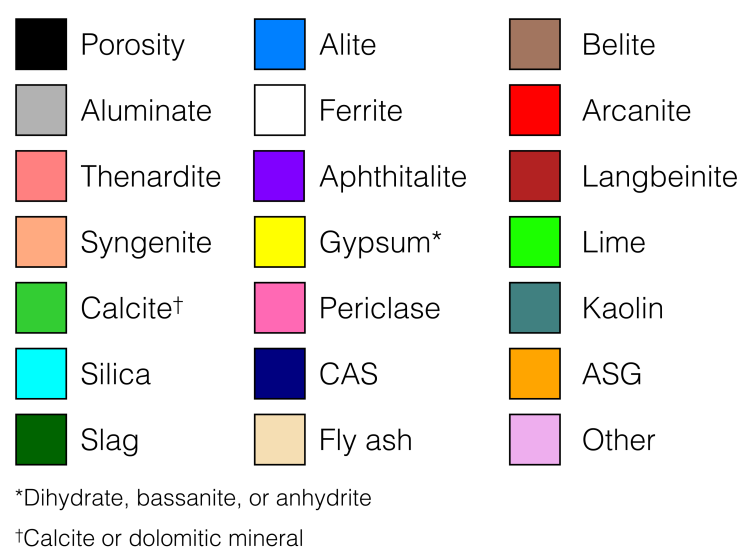

Additionally, if the Calculate Correlation Functions checkbox was activated, a collection of files, each ending with a different phase name, will be produced to store the two-point correlation function results for that phase.

\section{Calculation Principles and Algorithms}

This section briefly describes the principles used to perform the several image analyses described in the previous section.

\subsection{Volume Fraction}

The volume fraction ${ }^{\S}$ of each solid phase is measured by simple point counting of pixels. Let $N_{i}$ be the number of pixels of phase $i$ in a microstructure image having $n$ solid phases. Then the volume fraction $v_{i}$ on a total solids basis is

$$
v_{i}=\frac{N_{i}}{\sum_{k=1}^{n}\left(N_{k}\right.} \quad i=1,2, \ldots, n
$$

\subsection{Mass Fraction}

The mass fraction of each phase, on a total solids mass basis, is computed directly from the volume fractions by weighting each phase's volume fraction by its density. The densities assumed for each phase are shown

\footnotetext{
§Some confusion could arise over the use of the terms "volume" and "area" in image analysis. This document adopts the mathematical concept of volume as a measure of the size of a subspace having the same dimensionality as the parent space. Therefore, a volume in 3D space is a 3D quantity, and a volume in $2 \mathrm{D}$ (such as a microstructure image) is a 2D quantity. Likewise, area is the measure of a having dimension one fewer than the parent space. An area in 3D is a 2D quantity, and an area in 2D is a $1 \mathrm{D}$ quantity, which some might prefer to call a perimeter. However, we retain volume and area primarily because of their correspondence with volume and area of the actual 3D microstructure.
} 
Table 2: Density assumed for each solid phase.

\begin{tabular}{cc|cc}
\hline Phase Name & Density $\left(10^{3} \mathrm{~kg} \mathrm{~m}^{-3}\right)$ & Phase Name & Density $\left(10^{3} \mathrm{~kg} \mathrm{~m}^{-3}\right)$ \\
\hline Alite & 3.21 & Belite & 3.28 \\
Aluminate & 3.04 & Ferrite & 3.73 \\
Arcanite $_{\text {Gypsum }^{(\mathrm{a})}}$ & 2.66 & Thenardite & 2.68 \\
Calcite $^{(\mathrm{b})}$ & 2.32 & Lime & 3.31 \\
Silica $^{\text {CAS }}$ & 2.71 & Periclase & 3.78 \\
Other $^{(\mathrm{c})}$ & 2.65 & Kaolin & 2.60 \\
\hline
\end{tabular}

(a) "Gypsum" is used here as a generic name for the various hydrated and anhydrous calcium sulfate phases found in cementitious powders, and the assumed density is therefore an approximate value.

(b) "Calcite" as used here could include some magnesium impurities more properly identified as a dolomitic mineral, but the density assumed is for pure calcite.

(c) "CAS" represents a generic calcium aluminosilicate glass similar in composition to some glasses in fly ash materials; the density is an approximate value typical of aluminosilicate glasses.

in Table 2. The mass fraction, $m_{i}$, of phase $i$ is therefore

$$
m_{i}=\frac{\rho_{i} v_{i}}{\sum_{k=1}^{n}\left(\rho_{k} v_{k}\right.} \quad i=1,2, \ldots, n
$$

\subsection{Area Fraction}

The area fraction, $a_{i}$, of solid phase $i$ is defined here as the fraction of the combined interface area that has phase $i$ one one or the other side of it. For clinker images, an interface is the boundary between any two dissimilar phases. For cement powder images, an interface is the boundary between one solid phase and the interparticle porosity.

In a digitized image, interface area is measured by counting pixel edges. For example, a single square pixel of phase $i$ embedded in a different phase has an interface area of four edge lengths because the pixel has four edges. Therefore, the area fraction of phase $i$ is the sum of all the pixel edges of $i$ shared with another phase, divided by the sum of all the pixel edge lengths defining all the interfaces in the image,

$$
a_{i}=\frac{\sum_{j=1}^{N_{i}} L_{i j}}{\sum_{k=1}^{n}\left(\sum_{j=1}^{N_{k}} L_{k j}\right.}
$$

where $L_{i j}$ is the number of face edges of the $j$-th pixel of phase $i$ lying at an interface, and the denominator includes a sum over all $n$ phases in the microstructure image. 
The definition given in Eq. (3) effectively double-counts all the interface area because each edge at an interface is counted once for each of the two phases sharing that edge. This means that, for example, in a two-phase image, each phase is calculated to have an area fraction of 0.5 , even though each phase is present at all of the interface edges. One might argue that in such an image the area fraction should be 1.0 for both phases, but this convention would have the disadvantage of not being normalized to unity.

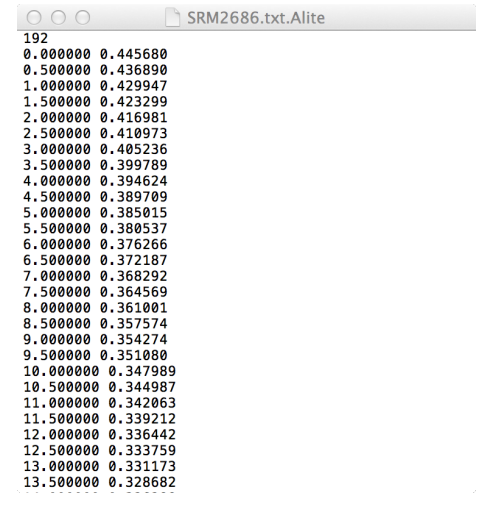

Correlation function data file

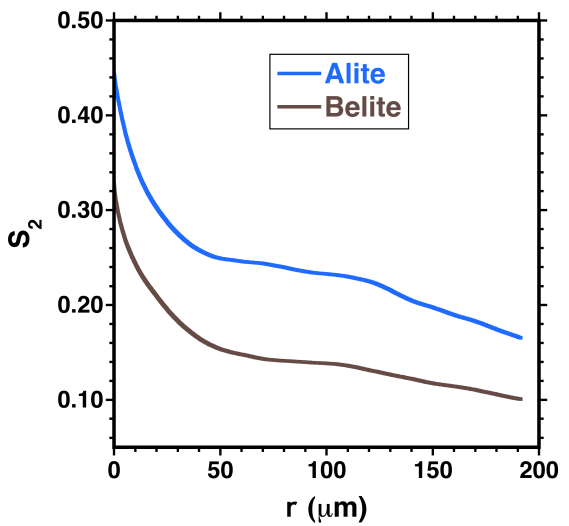

Plot

\subsection{Two-point correlation functions}

\subsubsection{Properties of two-point correlation functions}

The two-point correlation function for a phase, $S_{2}(r)$, is the probability that two randomly selected points in the image, separated by a distance $r$, both lie within that phase. In random composite materials, $S_{2}$ has some generic features that can be used to characterize the spatial distribution of the phase. For example, when $r=0$, the points coincide; the probability of a randomly selected point lying within a phase in a 2D image is equal to the area fraction, $\phi$, of that phase, on a total area basis. Therefore, $S_{2}(0)=\phi$, that is, the $y$-intercept of the plot is equal to the phase's area fraction. A second property of $S_{2}(r)$, which will not be proven here, is that its limiting slope as $r \rightarrow 0$ is directly proportional to the phase's boundary length per unit area, $\Sigma$. In particular, for granular media the exact relation can be shown to be [5]

$$
\lim _{r \rightarrow 0^{+}} \frac{d S_{2}}{d r}=-\frac{\Sigma}{4}
$$

A third property of $S_{2}$ is that, at sufficiently large distances between the two points in a random microstructure, the phase identity of each point should be independent of the other. In other words, the phase distribution should be uncorrelated at large distances. When two events are statistically independent, the joint probability is equal to the product of the (unconditional) individual event probabilities. As stated earlier, the probability of a single point lying within a phase is the phase's area fraction, $\phi$, so $S_{2}$ at sufficiently large distances should converge to $\phi^{2}$.

Examination of the plot below shows that $S_{2}(0)=0.4457$ for alite, which therefore is alite's area fraction on a total image area basis ${ }^{\mathbb{T}}$.

\footnotetext{
II Note that this fraction is somewhat lower than the value reported in the pfc data file for the same image because the latter value is normalized to the total solids area only.
} 


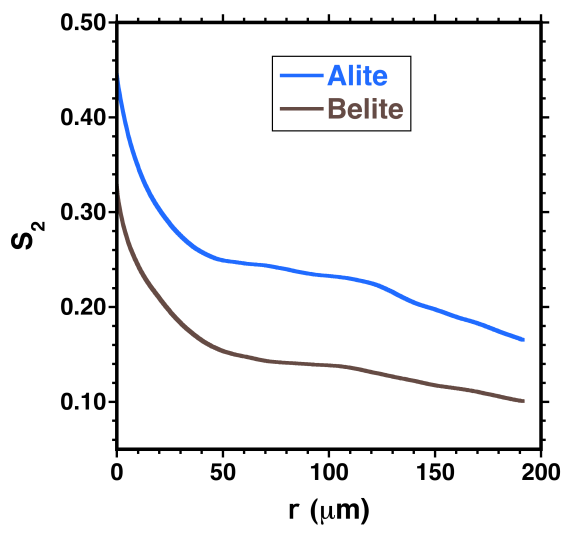

Furthermore, the slope at $r=0$ for alite is approximately $-0.0158 \mu \mathrm{m}^{-1}$, which provides a semiquantitative indication of the specific boundary area of alite in the image. Finally, the plot indicates that $S_{2}$ for neither alite nor belite appears to be converging to $\phi^{2}$, which is 0.198 for alite and 0.1024 for belite. This can be interpreted to mean that the spatial distribution of each phase is still somewhat correlated at distances as large as $200 \mu \mathrm{m}$.

The plot below shows the two-point correlation functions for the minority phases arcanite and periclase in a clinker material.

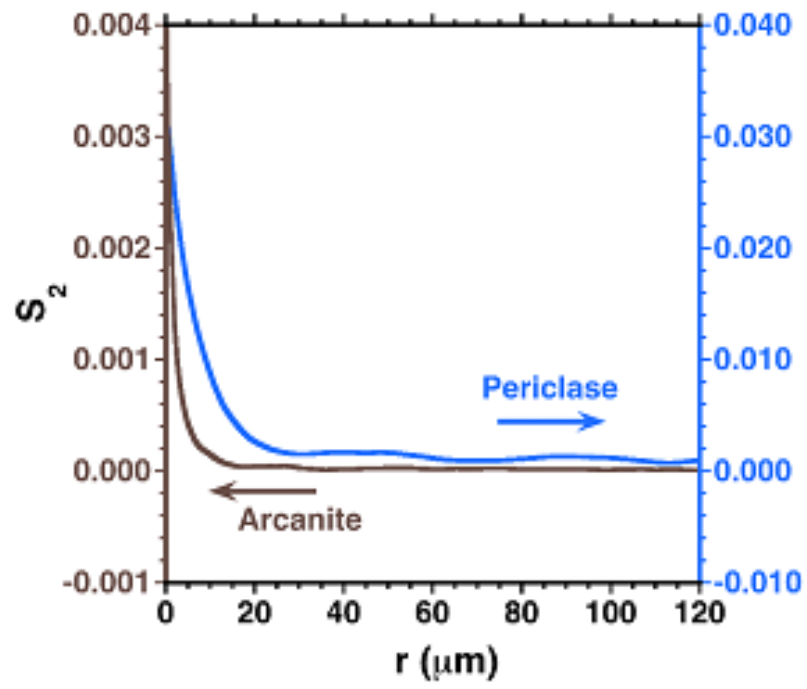

This plot highlights two other features of two-point correlation functions. First, the correlation functions appear to be converging to values of about $10^{-3}$ for periclase and about $10^{-4}$ for arcanite at distances no greater than about $100 \mu \mathrm{m}$. At $r=0$, the values of $\phi$ for periclase and arcanite are 0.0337 and 0.0046 , respectively. Squaring these values gives 0.0011 for periclase and 0.00002 for arcanite, both values of which are reasonably close to the long-distance values. Therefore, both periclase and arcanite are concluded to lose any spatial correlation at distances of about $100 \mu \mathrm{m}$.

The second feature of the two-point correlation functions in this plot is the appearance of a local minimum at short distances followed by a small-amplitude oscillation in the curves. The periodic local minima are characteristic of granular media and of non-overlapping domains in composites, such as periclase and arcanite. In such materials, the distance at which the first local minimum occurs corresponds to about 
half the average equivalent radius of the domain, and the distance at which the following local maximum happens corresponds to the average domain spacing in the microstructure [5]. Inspection of the plot suggests that the average equivalent radius of periclase and arcanite domains is about $31 \mu \mathrm{m}$ and about $18 \mu \mathrm{m}$, respectively. Likewise, the average domain spacings of periclase and arcanite are about $40 \mu \mathrm{m}$ and about $27 \mu \mathrm{m}$, respectively.

\subsubsection{Calculation of two-point correlation functions}

For a rectangular digitized images, the two-point correlation function can be conveniently calculated by convolution, by which, conceptually, two identical copies are overlaid and displaced relative to each other by a prescribed number of pixels in the $x$ direction and a prescribed number of pixels in the $y$ direction, and then counting the pixels that have the same phase located at the same position in the original image as in the displaced image [6, 7]. In an image of $M \times N$ pixels, the 2D two-point correlation function is

$$
S_{2}(x, y)=\sum_{i=1}^{M-x}\left(\sum _ { j = 1 } ^ { N - y } \left(\frac{I(i, j) I(i+x, j+y)}{(M-x)(N-y)}\right.\right.
$$

where $I(x, y)=1$ if the pixel at location $(x, y)$ contains the phase of interest and $I(x, y)=0$ otherwise. In a statistically isotropic microstructure, the $2 \mathrm{D}$ correlation function can be converted to be a $1 \mathrm{D}$ function of distance only $[6,7]$

$$
S_{2}(r)=\frac{1}{2 r+1} \sum_{i=0}^{2 r} S_{2} \quad r, \frac{\pi i}{4 r}
$$

where, for a given angle $\theta_{i}=\pi i / 4 r, S_{2}\left(r \cos \theta_{i}, r \sin \theta_{i}\right)$ is obtained by bilinear interpolation from the values of $S(x, y)$.

\section{Summary}

MicroChar is a straightforward application for performing quantitative phase analysis on indexed 2D images of cement powder or clinker microstructures. The kinds of data obtained from MicroChar can provide valuable information for monitoring and controlling the manufacturing of cement-based powders. The application can also facilitate the packaging of cement powder data in a form that can be uploaded to the VCCTL software package for simulating 3D cement microstructures, hydration, and engineering properties $[3,4]$.

\section{References}

[1] P. E. Stutzman, J. W. Bullard, and P. Feng. Quantitative Imaging of Clinker and Cement Microstructure. Technical Note. Washington, D.C.: National Institute of Standards and Technology, 2015.

[2] Imagef: Image Processing and Analysis in fava. Accessed: 2015-03-16. URL: http : / / imagej . nih . gov/ij/.

[3] VCCTL Software. Accessed: 2015-03-16. uRL: http: //www.nist.gov/el/building_materials/ evcct l. cfm. 
[4] Jeffrey W. Bullard. Virtual Cement and Concrete Testing Laboratory: Version 9.5 User Guide. Special Publication SP 1173. Washington, D.C.: National Institute of Standards and Technology, May 2014.

[5] J. G. Berryman and S. C. Blair. "Use of Digital Image Analysis to Estimate Fluid Permeability of Porous Materials: Application of Two-Point Correlation Functions”. In: J. Appl. Phys. 60.6 (1986), pp. 19301938.

[6] J. G. Berryman. "Measurement of Spatial Correlation Functions Using Image Processing Techniques”. In: F. Appl. Phys. 57.7 (1985), pp. 2374-2384.

[7] D. P. Bentz. "Three-Dimensional Computer Simulation of Portland Cement Hydration and Microstructure Development”. In: F. Am. Ceram. Soc. 80.1 (1997), pp. 3-21. 